\title{
Erratum to: Electronic CRM and perceptions of unfairness
}

\author{
Xiaoyu Yu • Bang Nguyen • Sung Ho Han • \\ Cheng-Hao Steve Chen $\cdot$ Fei Li
}

Published online: 30 January 2015

(c) Springer Science+Business Media New York 2015

\section{Erratum to: Inf Technol Manag \\ DOI 10.1007/s10799-014-0210-4}

The affiliation of one of the co-authors (Sung Ho Han) was incorrect in the original publication. The correct affiliation is shown here.

The online version of the original article can be found under doi:10.1007/s10799-014-0210-4.

\section{$\mathrm{X} . \mathrm{Yu}$}

School of Management, Shanghai University, Shanghai,

People's Republic of China

e-mail: yuxiaoyu@vip.126.com

\section{B. Nguyen ( $\square)$}

East China University of Science and Technology, School of Business, 130 Meilong Road, Xuhui District, Shanghai 200237,

People's Republic of China

e-mail: bang.london@gmail.com

\section{S. H. Han}

Faculty of Business, Oxford Brookes University,

Wheatley OX33 1HX, UK

e-mail: sunghohan7@hotmail.com

\section{C.-H. S. Chen}

Southampton University Business School, University of Southampton, Building 2, Highfield Campus,

Southampton SO17 1BJ, UK

e-mail: c.h.chen@soton.ac.uk

F. Li

Neeley School of Business, Texas Christian University,

Fort Worth, TX 76129, USA

e-mail: sabrina.li@tcu.edu 